\title{
CENITAL O El CAMINO HACIA EL CÉNIT DEL PETRÓleo
}

\author{
CENITAL, OR THE ROAD TO PEAK OIL
}

\author{
Montserrat López Mújica \\ Universidad de Alcala - Instituto Franklin
}

\section{Resumen}

En Cenital (2012), tercera entrega novelesca de Emilio Bueso, se nos presenta la visión distópica de un mundo en el que los combustibles fósiles han desaparecido. Las consecuencias ecológicas y sociales que esta situación conlleva incitan a la cruzada en contra del capitalismo salvaje y despiadado. Cenital es la cruenta y visionaria historia de una ecoaldea fortificada que se protege contra un mundo en el que las mascotas se han convertido en comida y el progreso es sólo el antepasado de la destrucción, la ruina y la barbarie. En este artículo realizamos una lectura ecocrítica de esta sorprendente y apocalíptica novela. Basándonos en la idea de la llegada de una nueva era, el Antropoceno, analizaremos cómo el colapso de la economía podría afectar al modo de pensar de todo un planeta.

Palabras clave: Antropoceno, Decrecimiento, Ecocrítica, Emilio Bueso, Humanidades Ambientales, Pico del petróleo.

\begin{abstract}
In Cenital (2012), the third novel of Emilio Bueso, we are presented with the dystopian vision of a world in which fossil fuels no longer exist. The ecological and social consequences this situation entails lead to a crusade against a savage and ruthless capitalism. Cenital is the cruel but visionary history of an eco-fortified village that protects itself against a world in which pets have become the food and progress is only the precursor of destruction, ruin, and barbarism. In this article we offer an ecocritical reading of this amazing and apocalyptic novel. Based on the idea of the arrival of a new era: the Anthropocene, we will discuss how the collapse of the economy may affect the mind-set of an entire planet.
\end{abstract}

Keywords: Anthropocene, Decrease, Ecocriticism, Emilio Bueso, Environmental Humanities, Peak Oil. 
La mano invisible te ha robado la cartera y el futuro, y no se detendrá cuando algunos gobernantes dimitan. Esto no se arregla con unos años de ajuste, ni inyectando capitales, ni nacionalizando bancos. Esto no se va a quedar en los aeropuertos sin aviones, los trenes de alta velocidad sin pasajeros, la gente sin pisos y los pisos sin gente. Esto solo se acabará cuando un silencio sepulcral se enseñoree de todas las grandes ciudades, cuando el apagón se vuelva permanente y las bicicletas se desplieguen por las autopistas de peaje.

Para entonces habrán muerto millones de personas (Cenital).

Parece que el viejo mundo que todos conocemos y en el que hemos habitado los últimos 12.000 años ha llegado a su fin (Davis, 2008). El periodo conocido con el nombre geológico de Holoceno (del griego holos, todo, y kainos, reciente: la era totalmente reciente) que coincide con el inicio de la agricultura y la expansión y evolución de las distintas civilizaciones humanas que conocemos en la actualidad, está dando sus últimos suspiros. En 1992, el periodista Andrew Revkin hizo ya una predicción:

Perhaps earth scientists of the future will name this new post-Holocene period for its causative element-for us. We are entering an age that might someday be referred to as, say, the Anthrocene. After all, it is a geological age of our own making'. Perhaps many readers ignored the minor linguistic difference and have read the new term as Anthro(po)cene! (Revkin, 2011).

De hecho, la palabra ganó una variante alternativa - Antropocenoacuñada por el biólogo Eugene Stoermer en los años 1980, siendo esta popularizada durante principios del 2000 por un ganador del premio Nobel, el químico Paul Crutzen. En 2008, la Comisión Estratigráfica de la Asociación Geológica de Londres comenzó el proceso para eventualmente adoptar el término "antropoceno" como nomenclatura oficial. Según una estimación realizada por un grupo de 30 expertos (Anthropocene Wor- 
king Group, AWG)', bajo la supervisión de la Comisión Internacional de Estratografía, el mayor grupo científico dentro de la Unión Internacional de Ciencias Geológicas (IUGS) reunidos en Berlín en el mes de octubre de $2014^{2}$, la Tierra ha entrado anticipadamente en el Antropoceno ("la era de los seres humanos") a causa de la acción humana y la alteración climática que sufre el planeta. Aunque como alternativa, también se considera que pueda ser una época menor en el nivel jerárquico, como una subdivisión del actual Holoceno. El Antropoceno es definido como la época geológica en la que una especie, el ser humano, ha demostrado ser capaz de alterar completamente el planeta: "I'homme est devenu une force géologique, et même sans doute la principale agissant aujourd'hui sur la terre" (Lourius \& Carpentier 2010:12).

Todo comenzó hace doscientos cincuenta años en Inglaterra. Impulsados por las energías fósiles como el carbón y el petróleo, surgen nuevos e innovadores inventos. Es lo que conocemos bajo el nombre de Revolución Industrial, que se extenderá rápidamente por todo el continente europeo y norteamericano, hasta llegar a Japón y a otras regiones del mundo. Las grandes infraestructuras de transportes (ferrocarril, carreteras) conectan a las personas por todo el mundo. Los grandes descubrimientos en la medicina salvan millones de vidas humanas. Los nuevos abonos artificiales que aparecen en el mercado posibilitan la alimentación de millones de personas. El crecimiento de la población en el planeta se dispara. Sin embargo, todo esto no es nada comparado con lo que todavía queda por venir: la "gran aceleración" comienza en la década de los cincuenta. La globalización, el marketing, el turismo y las grandes inversiones ayudan a alimentar este enorme crecimiento. Se produce un movimiento poblacional hacia las ciudades, que se convierten en los motores más potentes de la creatividad. En tan solo una generación, el "bienestar" de millones de personas mejora más allá de lo esperado (salud, dinero, seguridad, longevidad). Afortunadamente, no toda la especie humana actúa así, sino una parte cada vez más importante de la misma que se ve impulsada y condicionada por un sistema: el actual capitalismo, cuyos impactos en la sociedad y en los individuos, han logrado alterar por primera vez en la Historia el sistema ecológico y geomorfológico global (Fernández Duran, 2011 : 10). Nunca

1 El Grupo de Trabajo del Antropoceno es un grupo interdisciplinar de científicos y humanistas integrado por geólogos, científicos del clima, ecologistas y un abogado.

2 Este grupo de experto ha elaborado una propuesta que será analizada en el próximo Congreso Internacional de Geología, en 2016 
tantos tuvieron tanto, y sin embargo... casi mil millones de personas están desnutridas según la FAO en este planeta ${ }^{3}$. En una sola generación, el ser humano se ha convertido en una fuerza global fenomenal. Por poner algunos ejemplos, movemos más sedimentos y roca al año que todos los procesos naturales, como la erosión y los ríos del planeta en su conjunto. Utilizamos tres cuartas partes de toda la tierra firme que no está cubierta por glaciares. Hemos disparado los niveles de gas invernadero a épocas de hace más de un millón de años, con el consiguiente aumento de temperatura. Hemos provocado un inmenso agujero en la capa de ozono. Estamos perdiendo nuestra biodiversidad a pasos acelerados. Muchos de nuestros deltas se están literalmente hundiendo debido a la construcción de presas o a la minería. El nivel de nuestros océanos está subiendo imparablemente y su acidificación es una amenaza cada día más real. En una palabra, estamos alterando todos los ciclos naturales del planeta. "El siglo $\mathrm{XX}$, por tanto, es un fragmento diminuto, pero la escala de las transformaciones que ha presenciado empequeñece toda la historia humana anterior" (Christian, 2005).

Esta constante presión que el ser humano ejerce sobre el planeta está provocando una desestabilización sin precedente. Cada uno de nosotros forma parte de esta generación que, por primera vez, comprende la enorme responsabilidad que todo esto conlleva; y probablemente seamos también la última generación que tenga ocasión de remediarlo:

Una civilización se acaba y hemos de construir otra nueva. Las consecuencias de no hacer nada -o hacer demasiado poco- nos llevan directamente al colapso social, económico y ecológico. Pero si empezamos hoy, todavía podemos ser las y los protagonistas de una sociedad solidaria, democrática y en paz con el planeta (Manifiesto 2012: 3).

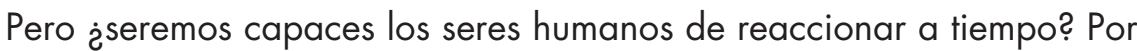
el bien de las generaciones futuras, necesitamos encontrar urgentemente un espacio operativo en el que la humanidad se sienta algo más segura. Un enorme reto se presenta en este contexto para las Humanidades, y particularmente, para la literatura. En este ámbito son muchas las voces de protesta que han surgido, a lo largo de estos últimos años, obligadas por una realidad ya demasiado visible: el cambio climático; estas voces tratan de concienciar al público de la necesidad de actuar inmediatamente y de manera global para frenar las malas decisiones políticas que se están lle- 
vando a cabo contra el medioambiente. Descatados investigadores ecocríticos como Scott Slovic o Kathleen Dean Moore proclaman que la literatura ayuda a difundir en las culturas qué es lo que está bien y lo que está mal, o a determinar qué es verdad (Moore, Slovic 2014: 3). La literatura nos ayuda además a crear a nuestro antojo cualquier realidad imaginable. Nos permite plantearnos preguntas y suscitar en el lector dudas sobre nuestro sistema actual en el que la economía está presente en todas las tomas de decisión, siempre bien apoyada por la industria del petroleo, y en especial en aquellas decisiones relacionadas con temas medioambientales. Podemos experimentar todo tipo de errores o catástrofes y prever lo que podría pasar, tal y como ocurre en la novela que analizaremos a continuación. A través de las obras de ficción el público puede llegar a vivir futuros completamente distintos a los nuestros, incluso en otros mundos; pero también experimentar cuales serían los resultados de continuar con nuestro actual ritmo de vida. Con esto, tanto los autores como los ecocríticos tratan de hacer recapacitar al lector sobre qué tipo de decisiones o medidas deberían llevarse a cabo para garantizar un futuro en el que seres humanos y no humanos por igual convivan de forma equilibrada.

En Cenital, Emilio Bueso realiza un acercamiento sorprendente al tema del cenit del petroleo ya que se centra en las probables consecuencias que puede acarrear a la humanidad continuar en esta peligrosa dirección. La catástrofe que transforma el mundo en Cenital tiene un origen estrictamente humano, por lo cual analizaremos la obra a la luz discursiva del contexto en que se produce, a saber, la sociedad posmoderna del capitalismo tardío, ese que según Destral, protagonista principal del relato, "nos tiene más absortos que una novia puta" y que su funcionamiento consiste en "crearte deseos absurdos e inalcanzables para que tú te desesperes tratando de satisfacerlos" (56). Nos hace pensar en un sombrío futuro en el que la humanidad tendrá que sobrevivir sin la principal fuente de energía que la ha hecho crecer durante todo el siglo XX. Sin lugar a duda, podemos referirnos a ella como una novela terrorífica por las enormes posibilidades que tiene de que todo lo que allí se narra pueda ocurrir en un futuro no muy lejano ${ }^{4}$. El autor se encarga además de darnos en el relato información de

4 Si tenemos en cuenta el informe sobre el Cenit de la producción mundial de petróleo realizado por Fernando Bullón Miró para la Asociación para el Estudio de los Recursos Energéticos (AEREN) los geólogos han estimado que la fecha del cenit de la producción mundial de petróleo tuvo lugar en la década pasada. Las estimaciones más fiables lo sitúan en algún momento entre los años 2004 y 2010. 
cada uno de los protagonistas y de su pasado. De este modo resulta más fácil para el lector sentirse identificado con alguno de ellos.

El relato de Cenital se desarrolla en tres tiempos narrativos bien diferenciados: el presente de la acción, alrededor del año 2014, el pasado anterior al desastre mismo y que podemos situar alrededor del año 2008 (año en el que comenzó la crisis), y las narraciones fragmentadas de los diferentes habitantes de la ecoaldea entre ambos periodos de tiempo, que varían desde historias personales enfocadas a retratar el sufrimiento y asfixia de la sociedad civilizada del siglo XXI, como la de Sapote o la de Nini, hasta narraciones muy cercanas temporalmente al presente referencial en donde el foco narrativo está en la descripción de la experiencia tras el desastre en su violencia más explícita, como en el caso de Saig'o. Estos capítulos de narración clásica con presentación de personajes se combinan además con las entradas en un blog ficticio y las citas sobre la crisis energética de personajes famosos, entre los que encontramos, destacados científicos, economistas, escritores, pero también citas de películas, de canciones o personajes del humor. Una cierta multidisciplinariedad aparece así en el relato.

En la narración de Destral, creador y líder de la ecoaldea, hallamos elementos claves como son la regresión social y el colapso de la civilización una vez acabados los suministros mundiales de petróleo. Pero quizás el gran acierto de Emilio Bueso es situar su relato en la España actual; describiendo paisajes reconocibles por todos y mezclando el 15-M, la burbuja inmobiliaria y la crisis financiera con un futuro inmediato en que los pocos supervivientes que lo pueblan han vuelto a las tradiciones más antiguas para poder sobrevivir. Este es el apocalipsis de Bueso, uno sobre el que no elabora demasiado en términos de causas, sino que se contenta con parafrasear a expertos de diferentes ámbitos -los "picoleros" (de la expresión anglosajona oil peak)- que, igual que su protagonista ficticio, profetizan un futuro cercano en donde el petróleo se agotará y el mundo entero se tornará en caos.

En el relato existe una clara intencionalidad utópica que efectivamente comienza a tomar forma en un proyecto social determinado lla ecoaldea "Cenital") varios años antes de que acontezca el desastre. La puesta en marcha de esta idea la lleva a cabo el protagonista de la historia, Destral, un visionario y antiguo ingeniero reconvertido en profeta del cataclismo y con posterioridad en líder consensuado del precario asentamiento que, al sentir que el final estaba cerca, crea una página web, http://www.cenital. net/, y desde ella anima a la gente a compartir con él un proyecto: poner en marcha un sistema productivo autosostenible y no dependiente de las energías fósiles con el siguiente mensaje: "[t]odavía podéis bajaros del 
mundo, podéis ser autosuficientes, cultivar vuestra propia comida, construir vuestra propia casa, hacer vuestro propio jabón, pan, ropa, riqueza", en definitiva, "constituir [una] comunidad al margen de la economía de mercado, al margen de la dinámica energética actual" (Bueso 49). En algunos lugares de España, ya se han desarrollado cooperativas de este tipo que permiten el autoabastecimiento local gracias a las energías renovables ${ }^{5}$, y todo ello a pesar de que nuestro ministerio de industria haga cuanto está en su mano por no favorecer su desarrollo. Este sencillo ejemplo indica que en lo local está el verdadero cambio, mientras las instituciones gubernamentales existentes se muestren impotentes para asumir sus responsabilidades.

La ecoaldea se sitúa en el interior de la comarca de Els Ports, en la frontera montañosa entre Teruel y Castellón, allí donde una vez se instalaron los maquis y todavía hay tierras sin explotar. En ella viven cien personas, acostumbradas al frío, a la comida escasa y a las privaciones, pero que debido a su capacidad de previsión no han tenido que recurrir al pillaje o al canibalismo, como saben que ocurre fuera de sus sólidos muros. Son personas solidarias, comprometidas con el mantenimiento de su forma de vida, cada una aporta conocimientos prácticos y/o alguna valiosa habilidad para la comunidad: agricultores, cocineros, médico, maestro, albanil, alfarero, forjador, zapatero, técnicos de aerogeneradores y artefactos movidos por energía solar, etc. Su día a día se encuentra repleto de carestía y trabajo de sol a sol en una vuelta al siglo XVIII sin resurgir posible. Este podría ser el resumen de la ecoaldea, lugar físico en el que trascurre la mayor parte de la novela.

La obra es también un alegato contra el colapso de un sistema agroalimentario insostenible que depende directamente del petroleo. Toda la infraestructura agraria come actualmente del petroleo. Destral nos recuerda: "[n]uestros campos de cultivo están tan industrializados y mecanizados que ya dependen íntegramente del gasoleo. Son fábricas de comida robotizada, no explotaciones agrícolas naturales" (Bueso 33). Esto significa que si el petroleo llegase a desaparecer o incrementara su precio excesivamente no habría forma de alimentar a toda la población. Y nos da un ejemplo : el precio del litro del petroleo impone los precios de la comida que acostumbramos a consumir. Desde los años ochenta del pasado siglo un kilo de buen arroz blanco envasado al vacio o un kilo de soja verde cuesta lo mismo que un litro de gasolina super. "Así que cuando el litro de combustible esté a tres o cuatro euros, el kilo de grano andará a la par, más o menos" (Bueso 34) ¿Cuándo tendrá lugar esta debacle? Dentro de cincuenta años 
o dentro de cincuenta días, no se sabe "lo que sí podemos hacer es comenzar a movernos" (34). Debemos ser conscientes de este "¿hipotético?" futuro y quizás defender un modo de sociedad más respetuosa con las personas y la tierra, apoyando el desarrollo de prácticas agrícolas respetuosas con el medio y preservando los recursos naturales, como nos recuerda el filósofo francés Pierre Rabhi, impulsor destacado del agro-ecologismo y del retorno a la tierra.

Otro de los temas que se critica también en la obra es la comercialización de los trasgénicos. Estos se están imponiendo poco a poco en todo el planeta y condicionan profundamente el funcionamiento de la obtención de semillas de manera natural, tal y como se ha hecho a lo largo de la historia, arruinando y marginando a millones de personas que dejan de ser autosuficientes en su alimentación; al tiempo que concentra las riquezas y el control de la producción de alimentos en un puñado de multinacionales tipo Monsanto. La actualidad está repleta de estos casos: países como Argentina o India se encuentran sumidos en este comercio con la soja y el algodón transgénico respectivamente. Los cultivos transgénicos suponen una vuelta de tuerca más en el impacto negativo de la agricultura industrial. En la novela, Destral y Agro mantienen una conversación sobre estas semillas transgénicas que han sido introducidas por error en los campos de la ecoaldea:

-Era trigo transgrénico, Destral. Semillas modificadas para resistir a las plagas y crecer en invierno. Para todo eso y para algo más.

Destral empezó a poner cara de pánico. Alzó sus cejas por toda respuesta.

- Para autodestruirse.

- Autodestruirse.

-Era un gen al que llamaban «terminator» - continuó diciéndole-. Recuerdo que en el 2006 trataron de insertarlo masivamente en todos los cultivos transgénicos para evitar la piratería.

- ¿Piratería...? ¿Qué piratería ni que demonios?

-Piratería, gran jefe. (Bueso 41)

Las semillas mencionadas con este gen Terminator son ya una realidad, aunque la idea parezca sacada de la peor película de terror. Tecnologías de Restricción en el Uso Genético (TRUGs) este es el nombre "oficial" de la tecnología Terminator que se conoce en Naciones Unidas y entre científi- 
cos. De comercializarse ${ }^{6}$, Terminator evitaría que los agricultores pudieran reutilizar su semilla a partir de sus cosechas, lo que los forzaría a recurrir al mercado de semillas comerciales. Bajo la escusa de que esta tecnología impediría la contaminación genética, empresas como Monsanto, DuPont y Syngenta, están negociando con los gobiernos en Naciones Unidas para que se levante la moratoria de facto sobre Terminator. En Cenital se nos advierte sobre esta nueva biotecnología y sus consecuencias: "Hemos sembrado grano, pero no simiente. Así que no habrá cosecha" (Bueso 42). Toda la sociedad debería movilizarse para parar el desarrollo de una tecnología que sólo persigue crear semillas suicidas, que no reporta ningún beneficio a la humanidad y que responde únicamente a los intereses de la industria biotecnológica, amenazando al mundo con una nueva Primavera Silenciosa. Contra esta iniciativa, la mundialmente conocida activista Vandana Shiva, lleva a cabo la campaña mundial de "Semillas de Libertad". En su Declaración de las semillas, Vandana advierte: "El caso más extremo de semilla no renovable es el de la tecnología Terminator, desarrollada con el objetivo de crear semillas estériles"7. La lucha de esta activista se centra en evitar que las grandes multinacionales controlen la vida de los agricultores a través de la venta forzosa de las semillas y de los pesticidas que lo acompañan. Porque todo esto conlleva a la pérdida irremediable de la soberanía alimentaria ${ }^{8}$. Los beneficios de la llamada 'revolución verde' -el empleo masivo de fertilizantes químicos, maquinaria pesada y semillas seleccionadas para incrementar la producción agrícola- y de la ingeniería genética para combatir problemas como la hambruna, no están dando los resultados esperados a largo plazo. El hambre no es un problema de falta de alimentación, sino de mal reparto de la riqueza. Si algún día los países pobres no tienen deuda externa y dedican sus terrenos para producir ali-

6 El Tratado de Libre Comercio entre EEUU y Europa que se está negociando en estos momentos abriría aún más las puertas de Europa a compañías como Monsanto.

7 Declaración de las semillas por Vandana Shiva http://www.varietatslocals.org/wp-content/ uploads/2012/10/Manifesto-Seed-Freedom.pdf

8 La soberanía alimentaria deberá surgir de nuevos modelos de agricultura que la humanidad necesitará para la transición hacia formas de agricultura que sean más ecológicas, biodiversas, locales, sostenibles y socialmente justas; estarán arraigadas en la racionalidad ecológica de la agricultura tradicional a pequeña escala, que representa ejemplos establecidos de formas acertadas de agricultura local. Tales sistemas han alimentado la mayor parte del mundo durante siglos y siguen alimentando a millones de personas en muchas partes del planeta $(M$. A. Altieri, 2004). 
mentos y no para vender productos a los ricos (té, cacao, café, algodón, cacahuetes...), entonces el hambre podría ser completamente erradicado. No escondamos los auténticos hechos para sentirnos mejor. Este es un tema que el mismo Papa Francisco ha señalado muy claramente en su reciente encíclica apuntando directamente a los responsables del actual sistema económico mundial:

La deuda externa de los países pobres se ha convertido en un instrumento de control, pero no ocurre lo mismo con la deuda ecológica. De diversas maneras, los pueblos en vías de desarrollo, donde se encuentran las más importantes reservas de la biosfera, siguen alimentando el desarrollo de los países más ricos a costa de su presente y de su futuro (punto 52).

Como ya hemos mencionado anteriormente, estamos viviendo un deterioro progresivo de la naturaleza que comenzó hace ya bastante tiempo, pero que se ha acentuado a partir de la segunda mitad del siglo XX. Nos hemos habituado a convivir con este deteriodo, de forma que no se acaba de percibir, sobre todo si no hay estadísticas ni informes que vengan de las instituciones para ver cómo se va descomponiendo el patrimonio natural. No podemos seguir mejorando nuestra calidad de vida destruyendo el entorno, el ambiente, los ecosistemas o la naturaleza. No podemos seguir creciendo indefinidamente en un mundo finito. Así nos lo recuerda Destral : "¿ ${ }^{T}$ Tiene algún sentido esto de que todos los pueblos aspiren a crear cada vez más riqueza, de forma indefinida, en un mundo finito que ya da signos inequívocos de agotamiento?" (Bueso 144) ¿̇Es tiempo de decrecer? "Haz como nosotros, apuesta por la simplicidad voluntaria" (Bueso 56). Emilio Bueso novela en Cenital los pensamientos de decrecimiento de Serge Latouche y de Carlos Taibo, como advirtiéndonos que estamos condenados a decrecer, digan lo que digan los figurantes que se supone que nos gobiernan o los vendedores de humo de los medios. Al sistema le sobra gente y anda en el proceso de expulsarla. Viviremos una contracción irremediable durante décadas, con millones de dramas humanos. El mensaje es claro: si no decrecemos voluntariamente decreceremos como en Cenital, no podemos seguir produciendo a costa de los recursos limitados del planeta, de los ciudadanos del Sur Global o incrementando el cambio climático. Destral nos reserva un "colapso súbito", el ocaso de los campeones, la catástrofe maltusiana. Algo que ya ocurrió hace quinientos años en la Isla de Pascua (Bueso 109) 9 . Decrecer es necesario y supone un cambio de

9 El geógrafo evolucionista Jared Diamond en su obra Colapso (2005) deduce que la causa última del colapso en la isla de Pascua no es biológica sino social. Lo que hace al sistema inviable y le fuerza a colapsarse no es la escasez de los recursos (según el argumento maltusiano) 
valores, nos dice Carlos Taibo en su libro En defensa del decrecimiento (Editorial Catarata). Estamos abogados a dirigirnos hacia un decrecimiento basado en la equidad y la redistribución de la riqueza. Este sería sin duda alguna el camino a tomar y así lo anuncia Destral en una de las publicaciones de su blog: "Soy el fundador y corresponsal de un grupo antisistema que está preparándose para decrecer de forma neoprimitivista, hacia una economía de subsistencia autosuficiente" (Bueso 95) ¿¿Debemos convertir la actual crisis de los mercados en una verdadera crisis del sistema para dar a luz un nuevo modelo de sociedad? ¿Sería posible una sociedad sostenible y ya no basada en el depredador capitalismo neoliberal, que de ciclo a ciclo y de burbuja en burbuja está conduciendo al planeta a un inminente colapso como el de la isla de Pascua, ahora masivamente amplificado a escala global?

Destral apuesta por la economía real, esa que sigue existiendo para quien esté dispuesto a apostar por ella, es decir, la agricultura "hay miles de fincas que necesitan agricultor" (Bueso 155), nos dice. Es otra de las fórmulas practicadas desde el decrecimiento: una forma de poner en práctica nuevas formas de economía, sostenibles, alternativas al capitalismo, que tengan que ver con la economía real, productiva, y que cuestionen el productivismo especulativo que está conduciendo al caos a la humanidad y al planeta. Estamos en un momento delicado, en el que el sistema capitalista está tratando, por todos los medios, de perpetuarse como modelo único, para seguir acelerando las desigualdades y la feroz acumulación de riqueza en unas pocas manos.

Sin embargo, el mundo utópico que se presenta en la ecoaldea de Cenital dista mucho de ser un modelo a seguir. Emilio Bueso describe un presente horrible y augura un futuro aún peor, una "edad oscura" y avisa: "[l]as edades oscuras como la que se cierne sobre Occidente suelen traer consigo el hambre, las guerras, el atraso y la despoblación. Millones de muertes, de malas migraciones, de vidas destrozadas" (Bueso 111). Nos muestra además una sociedad excesivamente patriarcal reinstalada en la

sino el exceso de su explotación, como un efecto sólo derivado de la escalada social de la competición. Los diversos clanes de Pascua se embarcaron en un juego colectivo de prestigio ostentoso donde todos pugnaban por superar a los demás en la erección de moaís, para lo que no dudaron en agotar el bosque del que extraían la madera para transportar las piedras a edificar. Y al escasear la madera dejaron de producir canoas con las que pescaban su principal fuente de proteínas. Pese a lo cual siguieron erigiendo moaís cada vez mayores hasta que ya no pudieron hacerlo más. Entonces los golpistas tomaron el poder, estalló la guerra civil y la isla de Pascua se desangró hasta extinguirse. 
barbarie, la insolidaridad, la explotación de animales y de humanos, en la que impera la ley del más fuerte ¿ $\mathrm{Se}$ trata más bien de una advertencia? El futuro que nos espera, si no reaccionamos a tiempo ¿̇será una involución de la sociedad actual que conocemos? Sin lugar a dudas, es una literatura incómoda, agresiva, que increpa, que hace crítica de nuestras costumbres y creencias, pero que perdura en la mente porque provoca desazón, y eso siempre es buena señal. Cenital es excelente, puesto que muestra sin vergüenza las partes desagradables de la supervivencia, las que se tienden a ignorar pero alrededor de las cuales tenemos nuestro sistema del bienestar.

\section{CONCLUSIÓn}

"El siglo XXI será el siglo más decisivo de la historia de la humanidad" (Manifiesto 2014: 2). La cuestión de la crisis ecológica debería ser el tema principal en todas las agendas políticas de todos los gobiernos porque como hemos visto y el propio Manifiesto nos advierte dicha cuestión "(...) determina todos los aspectos de la sociedad: alimentación, transporte, industria, urbanización, conflictos bélicos (...) estamos atrapados en la dinámica perversa de una civilización que si no crece no funciona, y si crece destruye las bases naturales que la hacen posible"(Manifiesto 2014: 3). Aunque "Cenital" sea una novela especulativa sobre un mundo futuro en el que no queremos creer, tenemos la obligación de vislumbrar y prever lo que el futuro podría depararnos si el petrolero y demás energías fósiles desapareciesen. La crisis económica que sirve de trasfondo en Cenital, y que termina en Apocalipsis está más cerca de lo que nos creemos o así pensamos una vez cerrada la última página del libro. Quizás estemos a tiempo de cambiar (algo) las cosas, antes de que las cosas nos cambien (del todo) a nosotros. Se trata pues de una obra imprescindible para despertar ante hechos que pueden estar a la vuelta de la esquina: nadie sabe si en unos años nos veremos inmersos en un paisaje devastado por la violencia y la falta de recursos, lamentándonos por no haber hecho caso a aquellos que nos lo advirtieron.

El tiempo parece agotarse. Apenas un lustro, leemos en el Manifiesto, "para asentar un debate amplio y transversal sobre los límites del crecimiento, y para construir democráticamente alternativas ecológicas y energéticas que sean a la vez rigurosas y viables. Deberíamos ser capaces de ganar grandes mayorías para un cambio de modelo económico, energéti$\mathrm{co}$, social y cultural. Además de combatir las injusticias originadas por el ejercicio de la dominación y la acumulación de riqueza, hablamos de un modelo que asuma la realidad, haga las paces con la naturaleza y posibilite la vida buena dentro de los límites ecológicos de la Tierra" (Manifiesto 
2012:3). El estilo de vida del siglo XXI tiene que avanzar por dos caminos: la eliminación casi total de los residuos y el fin de nuestra dependencia de los combustibles fósiles. Los líderes del G7 acaban de comprometerse, en junio del 2015, a que la economía global deje de depender de los combustibles fósiles - ¡para siempre! ìEs una pequeña esperanza o la evidencia de que el colapso está más cerca de lo que creemos? Es, sin lugar a dudas, un paso más hacia la esperanza que todo el mundo ha puesto en las resoluciones de la cumbre de París (diciembre 2015). ¿Podrá el planeta unirse con el objetivo de lograr un mundo sin combustibles fósiles? No nos quedan muchas oportunidades...!

Debemos intentar influenciar más en la humanidad con los estudios de sostenibilidad (un saber vivir dentro de los límites del planeta) y de equilibro natural, compaginados con aquellos otros que dotan a las mujeres y a los hombres de "equilibrio emocional y espiritual", me refiero a la Filosofía, la Historia, la Música, la Pintura o la Literatura. La ecocrítica estaría situada aquí como un nuevo campo disciplinario que rompe con la tradicional separación entre las ciencias y las letras, ya que se considera fundamental unir la visión de la naturaleza literaria con la científica y ecológica. Y la obra que hemos presentado es un claro ejemplo de cómo llevar a los lectores un tema tan importante como el problema de las energías fósiles.

\section{Bibliografía}

ALTIERI, M. A. (2004) "Linking Ecologists and Traditional Farmers in the Search for Sustainable Agriculture," Frontiers in Ecology and the Environment, 2, pp 35-42.

BUESO, E. (2012) Cenital. Editorial Salto de página, 278 pp.

Carta encíclica 'Laudato si' del Santo Padre Francisco sobre el cuidado de la casa común (2015)

http://ep00.epimg.net/descargables/2015/06/18/a039balc2b0e3a9d3d24380c8e762116.pdf

CHRISTIAN, D. (2005) Mapas del tiempo. Introducción a la gran historia. Critica, $720 \mathrm{pp}$.

DAVIS, M. (2008) "Bienvenidos al Antropoceno", www.sinpermiso.info DIAMOND, J. (2006) Colapso. Por qué unas sociedades perduran y otra desaparecen. Círculo de Lectores, $746 \mathrm{pp}$.

FERNANDEZ DURÁN, R. (2011) El Antropoceno. La Expansión del capitalismo global choca con la biosfera. Virus editorial, 103 pp.

HEISE, U. K. (2013) "Globality, Difference, and the International Turn in Ecocriticism," MLA 128, 3, pp 642. 
LORIUS, C. \&CARPENTIER, L. (2010) Voyage dans l'Anthropocène. Cette nouvelle ère dont nous sommes les héros. Actes Sud, $200 \mathrm{pp}$.

MOORE, D. \& SLOVIC, S. (2014) "Editor's Note". ISLE: Interdisciplinary Studies in Literature and Environment, 21, 1 (Winter), pp 1- 4.

RABHI, P. (2008) Manifeste pour la terre et l'humanisme. Actes Sud, 124 Pp.

REVKIN, A. (1992) Global Warming: Understanding the Forecast. Abbeville $\mathrm{Pr}$; 1 st Edition Stated edition.

-, Confronting the 'Anthropocene' The New York Times, may 11, 2011. http://dotearth.blogs.nytimes.com/2011/05/11/confronting-the-anthropocene/?_r=0

Última llamada (Manifiesto) Estamos ante una crisis... de civilización (2014) https://ultimallamadamanifiesto.wordpress.com/el-manifiesto/ 